\title{
Efficacy of polyethylene glycol-propylene glycol- based lubricant eye drops in reducing squamous metaplasia in patients with dry eye disease
}

This article was published in the following Dove Press journal:

Clinical Ophthalmology

\author{
Alejandro Aguilar ${ }^{1,2}$ \\ Martin Berra ${ }^{3}$ \\ Jorge Trédicce 4 \\ Alejandro Berra ${ }^{5}$ \\ 'Department of Ophthalmology, \\ Medical Group Las Lomas, Buenos \\ Aires, Argentina; ${ }^{2}$ Department of \\ Ophthalmology, University of Salvador, \\ Buenos Aires, Argentina; ${ }^{3}$ Department \\ of Ophthalmology, Lagleyze Hospital, \\ Buenos Aires, Argentina; ${ }^{4}$ Department \\ of Ophthalmology, Santa Lucia \\ Hospital, Buenos Aires, Argentina; \\ ${ }^{5}$ Department of Pathology, University \\ of Buenos Aires, Buenos Aires, \\ Argentina
}

Purpose: Squamous metaplasia in dry eye disease (DED) manifesting as the loss of conjunctival goblet cells results in reduced mucin secretion and tear film instability. The objective of this study was to evaluate the efficacy of a polyethylene glycol-propylene glycol/hydroxypropyl-guar (PEG-PG/HP-guar) artificial tear formulation in reducing the squamous metaplasia in patients with DED using conjunctival impression cytology (CIC).

Methods: In this Phase IV, single-arm, open-label study, DED patients (aged $\geq 18$ years) with a corneal staining sum score $\geq 3$ and tear film break-up time (TFBUT) $<7$ s self-administered the PEG-PG/HP-guar artificial tears, 3 times a day for a period of 90 days. The primary end point was the change from baseline in goblet cell density (Nelson's CIC grading score) over the treatment period. Other end points were change in the corneal and conjunctival staining scores, and TFBUT. Statistical evaluation was performed using a paired $t$-test.

Results: In total, 49 patients ( $\mathrm{n}=98$ eyes) completed the study. Compared with baseline, there was a significant reduction in the mean CIC scores (ie, improvement in goblet cell density) at Days 30, 60, and 90 (1.6 \pm 0.5 vs $1.2 \pm 0.5,0.9 \pm 0.5$, and $0.8 \pm 0.5 ; P<0.0001)$. At Day $90,22 \%$ of eyes demonstrated squamous metaplasia Grade 0 (ie, normal epithelium). Similar improvements were observed in the corneal staining scores ( 5.7 vs $3.1,1.1$, and $0.5 ; P<0.0001$ ), conjunctival staining scores (5.5 vs 3.6, 1.6, and $0.9 ; P<0.0001$ ), and TFBUT ( 4.8 vs 5.8, 6.3, and $6.8 \mathrm{~s}$; $P<0.0001)$ at Days 30,60, and 90, respectively.

Conclusion: In this study, treatment with PEG-PG/HP-guar artificial tears for 90 days decreased CIC score, reduced corneal and conjunctival staining, and increased TFBUT in patients with DED. These results suggest that PEG-PG/HP-guar artificial tears can improve the ocular surface health and reverse the changes induced by squamous metaplasia in DED.

Keywords: artificial tears, hydroxypropyl-guar, squamous metaplasia, dry eye disease

\section{Introduction}

The epithelial cells of the cornea and conjunctiva are nonkeratinized, stratified squamous cells that secrete glycosylated glycoproteins called mucins. ${ }^{1-4}$ Mucins are essential to maintain tear film integrity; they form the glycocalyx that helps the aqueous layer to adhere to the hydrophobic cornea, acts as a lubricant, and forms a mucosal barrier. ${ }^{1,2,4}$ Interspersed within the conjunctival epithelia are goblet cells, which are specialized epithelial cells. These are the only cells that secrete the gel-forming mucin MUC5AC, which plays an important role in ocular surface protection and prevents it from desiccation. ${ }^{1,2,4}$ Morphological changes, loss of goblet cells, or alteration in their function thus have an adverse impact on the tear film and the ocular surface. ${ }^{1-4}$
Correspondence: Alejandro Aguilar Department of Ophthalmology, University of Salvador, Tucumán 1699 , Cl050AAG, Buenos Aires, Argentina Tel/fax +54 9I I 47354004

Email aguilar.alejandrojavier@usal.edu.ar (c) (1) (8) 2018 Aguilar et al. This work is published and licensed by Dove Medical Press Limited. The full terms of this license are available at https://www.dovepress.com/terms.php cc) ${ }_{\mathrm{BY}} \mathrm{NC}$ and incorporate the Creative Commons Atribution - Non Commercial (unported, v3.0) License (http:///creativecommons.org/licenses/by-nc/3.0/). By accessing the work you hereby accept the Terms. Non-commercial uses of the work are permitted without any further permission from Dove Medical Press Limited, provided the work is properly attributed. For permission for commercial use of this work, please see paragraphs 4.2 and 5 of our Terms (https://www.dovepress.com/terms.php). 
Dry eye disease (DED) is an ocular surface disorder characterized by tear hyperosmolarity and instability, which triggers a self-perpetuating chain of events that leads to dryness, cytokine-mediated inflammation and infiltration, an altered lacrimal function, and ocular surface damage., In DED, damage to the cornea and conjunctiva is manifested as squamous metaplasia, a pathological condition characterized by a nonsecretory, keratinized epithelium and loss of goblet cells. In DED, the goblet cell loss results in mucin deficiency which causes further tear film instability. ${ }^{4}$ A decrease in MUC5AC glycoprotein expression is observed as the dry eye condition progresses. ${ }^{7}$ Further, the degree of squamous metaplasia has been shown to correlate with the severity of DED. ${ }^{8}$

Systane ${ }^{\circledR}$ Ultra (Alcon Research Ltd, Fort Worth, TX, USA) is an artificial tear formulation containing the viscoelastic agent hydroxypropyl-guar (HP-guar), demulcents polyethylene glycol (PEG) and propylene glycol (PG), sorbitol, and borate preserved in Polyquad ${ }^{\circledR}$.,10 These PEG-PG/ HP-guar lubricant eye drops are indicated for symptomatic relief and extended ocular surface protection in patients with DED. The efficacy and safety of PEG-PG/HP-guar artificial tears in patients with DED have been demonstrated in clinical studies. ${ }^{9-15}$ However, the effect of these lubricant eye drops on goblet cell density in DED has not been studied.

The aim of this study was to evaluate the efficacy of PEG-PG/HP-guar artificial tears in reducing squamous metaplasia in patients with $\mathrm{DED}$, as assessed by conjunctival impression cytology (CIC).

\section{Methods}

This was a 3-month, prospective, Phase IV, single-center, open-label, single-arm study conducted from December 2009 to September 2010, at the Department of Ophthalmology, Medical Group Las Lomas, San Isidro, Buenos Aires, Argentina. Following screening (Day 0), eligible patients with DED were to instill 1 drop of the PEG-PG/HP-guar lubricant eye drops 3 times a day for a period of 90 days. Both eyes of patients were included. Patients had follow-up visits at Days 30, 60, and 90 .

Patients were assigned a study number in numerical sequence. The test lubricant drops were provided by the designated study staff. All patients provided written informed consent before enrollment into the study. The study was approved by the Ethics Committee-Teaching and Research Committee of Medical Group Las Lomas and was conducted in accordance with the Declaration of Helsinki and Good Clinical Practices. The study was registered with the National
Administration of Drugs, Foods, and Medical Devices in compliance with the local regulatory requirements of Argentina.

\section{Patients}

Male and female patients aged $\geq 18$ years who had active signs and symptoms of dry eye as determined by the study investigator, a sodium fluorescein corneal staining sum of $\geq 3$ in either eye, a tear film break-up time (TFBUT) of $<7 \mathrm{~s}$, and a CIC of Grade I to Grade III were considered eligible to participate in the study.

Patients were ineligible if they had/met any of the following conditions: 1) had punctal occlusion of any type, 2) had any severe or serious ocular or other medical condition, 3) were hypersensitive to the test product or any excipients, 4) were unwilling to discontinue contact lens wear at least 7 days prior to the screening visit or during the study period, 5) had a history of ocular trauma in either eye within 3 months prior to the screening visit or any ocular surgery within 6 months prior to the screening visit or planned surgery during the study period, 6) had any ocular infection or a history of ocular herpes, 7) were using either $\beta$-blockers or any other drug with anticholinergic side effects or any other topical ocular medication, 8) were using any systemic medication that could contribute to dry eye (including, but not limited to, cold and allergy treatments, tricyclic antidepressants, and hormone replacement therapies) and had not been on a stable dose for at least 30 days prior to the screening visit or had any anticipated change in dosing regimen during the course of the study, or 9) participated in any other investigational clinical study within 30 days prior to the screening visit.

\section{Study end points}

The primary end point of the study was the change from baseline in goblet cell density (determined by CIC) over the 3-month treatment period. Exploratory end points were the change from baseline in 1) total corneal staining score, 2) total conjunctival staining score, 3) TFBUT at Days 30, 60 , and 90, and 4) adverse events (AEs).

\section{Assessments}

All efficacy assessments were performed at Day 0 (baseline) and at Days 30, 60, and 90 of the study. Impression cytology specimens were obtained from different areas of the conjunctiva of each patient. Briefly, after topical anesthesia Propacaína (Anestalcon, Alcon Laboratories, Inc., Fort Worth, Texas, USA) in the inferior sac, semicircular filters (polyvinylidene fluoride filter, $\sim 15 \mathrm{~mm}$ diameter, $22 \mu \mathrm{m}$ pore; EMD Millipore, Billerica, MA, USA) were applied to the 
Table I Nelson's grading system for impression cytology

\begin{tabular}{|c|c|c|}
\hline Grade $^{\mathrm{a}}$ & $\begin{array}{l}\text { Goblet cells } \\
\text { characteristics }\end{array}$ & Epithelial cells characteristics \\
\hline 0 & Normal density & Normal cells \\
\hline I & Decreased density & $\begin{array}{l}\text { Reduced nucleocytoplasmic ratio } \\
\text { ( } 1: 3 \text { ratio) }\end{array}$ \\
\hline II & Absence of cells & $\begin{array}{l}\text { Larger cells with a nucleocytoplasmic } \\
\text { ratio of } 1: 4 \text { to } 1: 5\end{array}$ \\
\hline III & $\begin{array}{l}\text { Absence of cells plus } \\
\text { squamous metaplasia }\end{array}$ & $\begin{array}{l}\text { Cells with a large eosinophilic } \\
\text { cytoplasm with folded edges and } \\
\text { pyknotic nuclei (nucleocytoplasmic } \\
\text { ratio of more than } 1: 6 \text { ) }\end{array}$ \\
\hline
\end{tabular}

Notes: a A combination of grades (eg, Grade I-II, Grade II-III, Grade I-III, and so on) can be present at the same time in the cytological sample of a patient. When improving, the cellular changes progress following the same pattern (Grade III pass to Grade II, Grade II pass to Grade I, and Grade I pass to Grade 0), in the same sample from the same patient.

inferior tarsal and bulbar conjunctiva for $\sim 10 \mathrm{~s}$. The filters were peeled off and immersed in absolute ethanol for fixation. The specimens were rehydrated in 70\% ethyl alcohol and placed successively in periodic acid-Schiff (PAS) reagent, sodium metabisulfite, Gill's hematoxylin, and Scott's tap water. The specimens were rinsed with 95\% alcohol and absolute alcohol. The filter papers were made transparent with xylene, mounted on glass slides, and examined under a conventional light microscope (400× magnification). The specimens were graded using the Nelson grading system (Table 1). The point-counting technique was used for morphometric analysis, where PAS-positive areas were counted across 15 random microscopic high-power fields on a 100 point and 50-line grid on a video system coupled to the microscope. For each subject, an average of the 15 fields was taken. All the specimens were analyzed by the same investigator who was masked to the origin of the samples. Sample CIC images showing epithelial and goblet cell morphology in normal and severe dry eye are shown in Figure 1.

For TFBUT, $2 \%$ sodium fluorescein $(1-5 \mu \mathrm{L})$ was instilled onto the bulbar conjunctiva. The subject was instructed to blink several times, without squeezing, to distribute the fluorescein. Thereafter, the subject was asked to stare straight ahead without blinking, and the examiner observed the eye through a set slit-lamp (10× magnification) using cobalt blue light; a Wratten \#12 yellow filter was used to enhance observation of the tear film over the entire cornea.

TFBUT measurements preceded the corneal staining. The degree of corneal fluorescein staining was measured with a slit-lamp, for each of the 5 regions of the cornea (central, nasal, temporal, superior, and inferior) on a scale ranging from 0 (no staining) to 3 (severe staining). The scores of the 5 areas were summed to obtain the total score for each eye, resulting in a scale from 0 (no staining in any area) to 15 (severe staining in all the areas).

For conjunctival staining, $5 \mu \mathrm{L}$ of Lissamine Green was instilled in each eye, and the staining was examined with a slit-lamp for each of the 6 regions (proximal superior, proximal inferior, and distal regions of both temporal and nasal conjunctiva). Each region was graded on a scale of 0 (none) to 3 (severe). The scores of the 6 areas of the conjunctiva were summed for each eye, resulting in a scale from 0 (no staining in any region) to 18 (severe staining in all regions).

Data on AEs, self-reported or observed, were collected at each visit and recorded in an $\mathrm{AE}$ form. For each AE, the severity (mild, moderate, or severe) and the causality were determined by the investigator.

\section{Statistics}

Statistical analyses were performed using SAS software. A sample size of 50 patients was planned to be enrolled. The differences in mean values between the baseline and subsequent visits following treatment were estimated within \pm 0.40 standard deviation (SD) using a 95\% 2-sided confidence interval, based on repeated analysis of variance.

For all outcomes, paired $t$-tests were conducted to compare baseline vs outcomes at Days 30, 60, and 90.

\section{Results}

Of the 50 patients enrolled, 49 ( $n=98$ eyes) completed the study at Day 90 . One patient discontinued the study due to
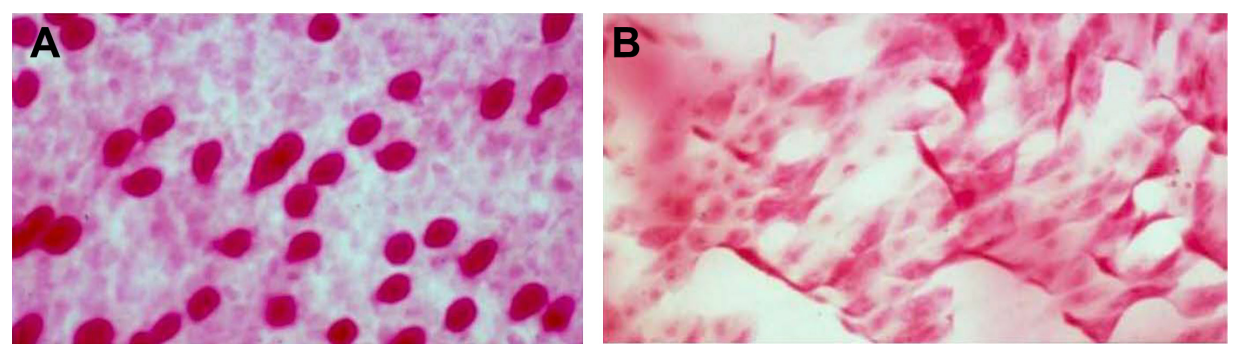

Figure I Representative PAS-and $\mathrm{H}$ and E-stained images at 400× magnification showing (A) a normal number and morphology of goblet cells (Grade 0) (B) squamous metaplasia (Grade 3). 


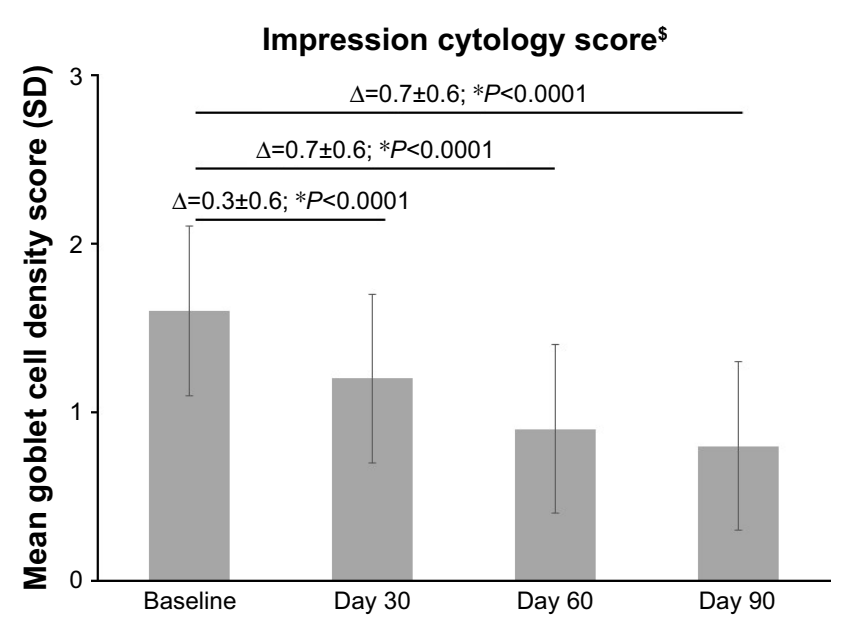

Figure 2 Mean conjunctival impression cytology scores (ie, mean goblet cell density) at baseline and Days 30, 60, and 90 after treatment with PEG-PG/HP-guar artificial tears.

Notes: ${ }^{* P}$-value for mean change in scores vs baseline. ${ }^{\$}$ The lower the scores, the greater the improvement.

Abbreviations: PEG-PG/HP-guar, polyethylene glycol-propylene glycol/hydroxypropyl-guar; SD, standard deviation.

a nonserious AE (intolerance to the test formulation). The patients were aged between 21 and 70 years; $90 \%(n=45)$ of patients were female.

\section{Primary end point}

There was a significant improvement in CIC score at all follow-up time points compared to baseline $(P<0.0001$; Figure 2). The mean \pm SD cytology score decreased from
$1.6 \pm 0.5$ units at baseline (range, $0-3$ ) to $1.2 \pm 0.5$ units at Day 30, 0.9 \pm 0.5 units at Day 60, and $0.8 \pm 0.5$ units (range, 0-2) at Day 90 (Figure 2).

At baseline (Figure 3A), 6.1\% of eyes $(n=6 / 98)$ were classified as having abnormal CIC Grades II-III, 25.5\% of eyes $(n=25 / 98)$ as Grade II, $36.7 \%$ of eyes $(n=36 / 98)$ as Grades I-II, and 15.3\% ( $\mathrm{n}=15 / 98)$ of eyes as having Grade I severity. By Day 90, the severity of squamous metaplasia reduced in most eyes; $22 \%$ of eyes $(n=22 / 98)$ had improved to Grade 0 (ie, normal morphology), whereas $64 \%$ of eyes $(n=63 / 98)$ were classified as Grade I; only 3 eyes were classified to have Grade II severity (Figure 3B). No change in grade was observed for $8.2 \%$ of eyes $(n=8 / 98)$ during the study.

\section{Exploratory end points}

The cornea and conjunctival staining scores and TFBUT improved over the duration of the study. As shown in Figure 4, there was a marked decrease in the corneal staining sum score from baseline to Days 30, 60, and $90(P<0.0001)$. The mean $\pm \mathrm{SD}$ corneal staining score improved from $5.7 \pm 2.8$ units (range, $3-11$ ) at baseline to $3.1 \pm 2.3$ units at Day 30 (range, 0-8), 1.1 \pm 0.9 units at Day 60 (range, 0-3), and $0.5 \pm 0.6$ units (range, $0-2$ ) at Day 90. All eyes demonstrated a total staining sum score $<3$, with $59 \%$ of eyes $(n=58 / 98)$ having a staining sum score of 0 at Day 90.

\section{A Impression cytology grade \\ Baseline}

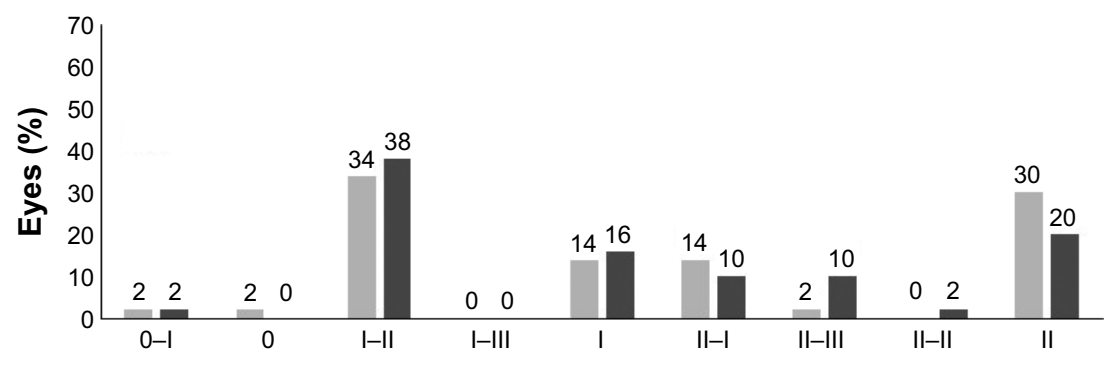

B

Day 90

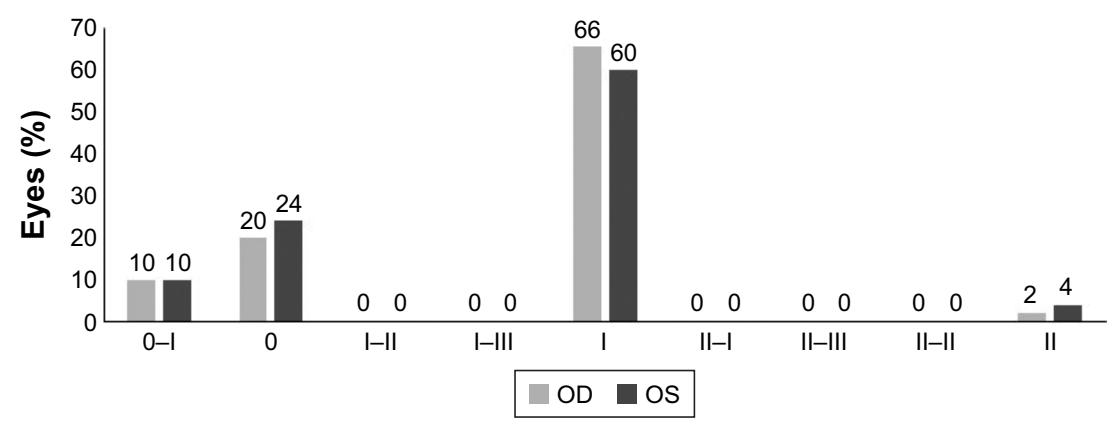

Figure 3 Conjunctival impression cytology grades of eyes at (A) baseline and (B) 90 days after treatment with PEG-PG/HP-guar artificial tears. Abbreviations: OD, oculus dextrus (right eye); OS, oculus sinister (left eye); PEG-PG/HP-guar, polyethylene glycol-propylene glycol/hydroxypropyl-guar. 


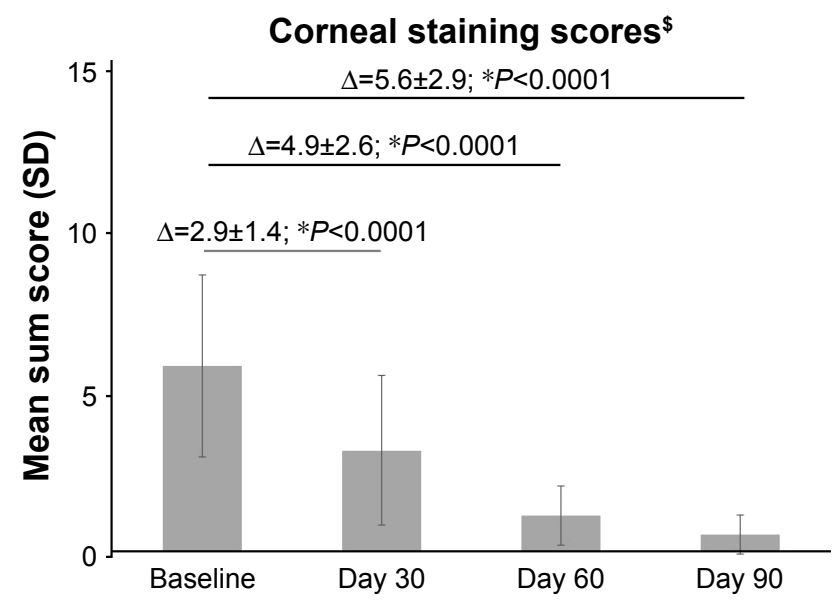

Figure 4 The mean corneal staining sum score at baseline and Days 30, 60, and 90 after treatment with PEG-PG/HP-guar artificial tears.

Notes: *P-value for mean change in scores vs baseline. ${ }^{\$}$ The lower the scores, the greater the improvement.

Abbreviations: PEG-PG/HP-guar, polyethylene glycol-propylene glycol/ hydroxypropyl-guar; SD, standard deviation.

Compared with baseline, there was a notable decrease in the mean \pm SD conjunctival staining score at all follow-up visits $(P<0.0001$; Figure 5$)$. The mean conjunctival staining scores improved from 5.5 \pm 2.1 units (range, $3-11$ ) at baseline to 3.6 \pm 2.0 units at Day 30 (range, $0-9$ ), $1.6 \pm 1.1$ units at Day 60 (range, $0-5$ ), and $0.9 \pm 0.9$ units (range, $0-3$ ) at Day 90 .

At the baseline, the mean \pm SD TFBUT was $4.8 \pm 1.0 \mathrm{~s}$ (range, 3-6), and increased to $5.8 \pm 1.0 \mathrm{~s}$ (range, 3-8) at Day 30 and $6.3 \pm 0.9 \mathrm{~s}$ (range, $4-8$ ) at Day 60 , ending at $6.8 \pm 0.9 \mathrm{~s}$ (range, 5-9) at Day $90(P<0.0001$ vs baseline at all time points; Figure 6). At Day 90, almost $61 \%$ of patients reached a TFBUT of $\geq 7 \mathrm{~s}$ vs none at baseline.

One patient discontinued the study due to a nonserious $\mathrm{AE}$ (intolerance to the test formulation). No other AEs were reported in the study.

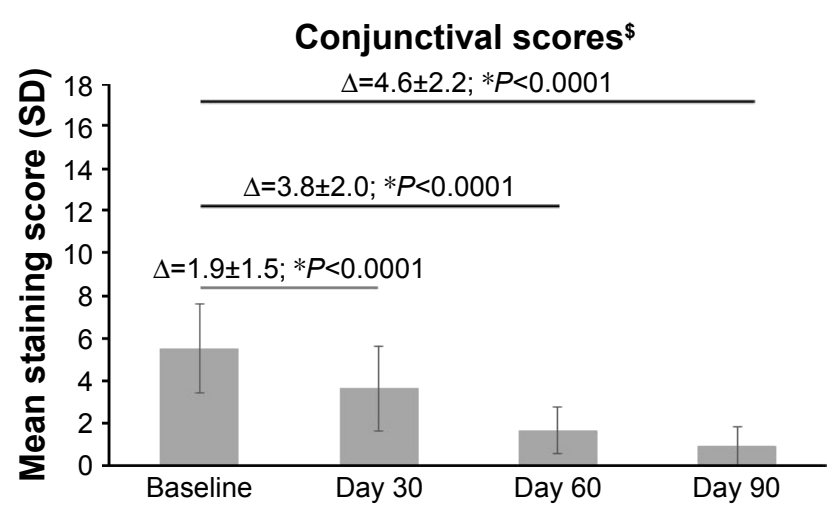

Figure 5 The mean conjunctival staining score at baseline and Days 30, 60, and 90 after treatment with PEG-PG/HP-guar artificial tears.

Notes: $* P$-value for mean change in scores vs baseline. ${ }^{\$}$ The lower the scores, the greater the improvement.

Abbreviations: PEG-PG/HP-guar, polyethylene glycol-propylene glycol/ hydroxypropyl-guar; SD, standard deviation.

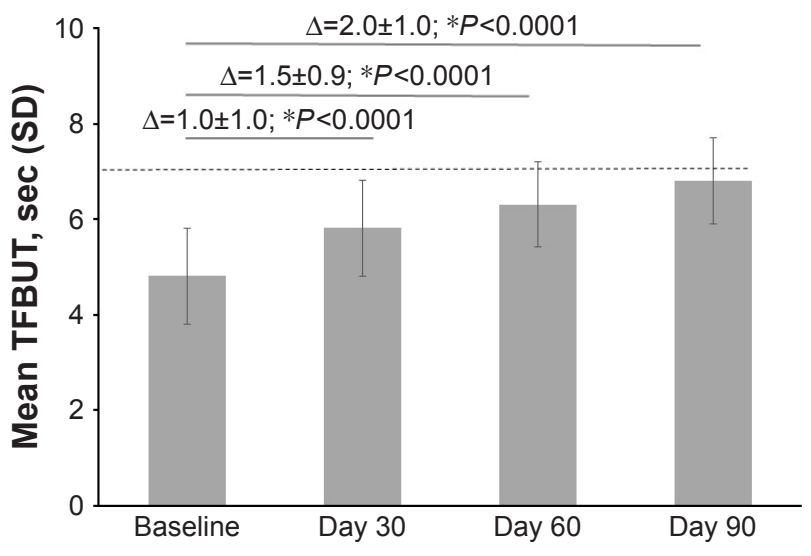

Figure 6 Mean TFBUT at baseline and Days 30, 60, and 90 after treatment with PEG-PG/HP-guar artificial tears.

Notes: $* P$-value for mean change in scores vs baseline. Dashed lines indicate the inclusion criterion at baseline (TFBUT $<7 \mathrm{~s}$ ).

Abbreviations: PEG-PG/HP-guar, polyethylene glycol-propylene glycol/ hydroxypropyl-guar; SD, standard deviation; TFBUT, tear film break-up time.

\section{Discussion}

This study demonstrated that the use of PEG-PG/HP-guarbased artificial tears can help in reversing the changes induced by squamous metaplasia in DED. After 3 months of treatment, a $50 \%$ decrease in the squamous metaplasia score (ie, an increase in goblet cell density) was observed compared with baseline, the majority of eyes ( $92 \%)$ demonstrated an improvement in the Nelson's impression cytology grade, and $22 \%$ of eyes reached Grade 0 (ie, normal epithelial morphology and $>500$ goblet cells $/ \mathrm{mm}^{2}$ ).

Similar improvements were observed in other signs of DED. There was an approximate $92 \%$ and $74 \%$ reduction in the mean corneal staining sum score and conjunctival staining sum score from baseline by Day 90, respectively. An increase of $2 \mathrm{~s}$ was observed in the mean TFBUT at Day 90. Both corneal and conjunctival stainings are indicative of a compromised ocular surface, and patients with DED have a faster TFBUT than normal individuals due to an unstable tear film. These results show that PEG-PG/HP-guar artificial tears improve ocular surface health and enhance the tear film stability in DED, which is in agreement with the outcomes reported with HP-guar-containing artificial tears in the literature. ${ }^{9-19}$

The observed therapeutic effect with PEG-PG/HP-guar lubricant eye drops is most likely due to the unique gelling ability of HP-guar in situ. When exposed to tear film $\mathrm{pH}$, HP-guar together with borate ions forms a soft gel-like cross-linked matrix on the innermost mucin layer, allowing longer retention of the demulcents PEG and PG on the ocular surface..$^{9,10,16}$ This in turn results in longer-lasting hydration and lubrication on the ocular surface and provides a conducive environment for healing and renewal of 
the damaged goblet and epithelial cells., ${ }^{9,10,16}$ Alternatively, a significant reduction in the expression of inflammatory marker human leukocyte antigen-DR has been observed in DED patients with regular use of PEG-PG/HP-guar eye drops. ${ }^{20,21}$ Squamous metaplasia and loss of goblet cells in DED are considered to be the consequences of inflammation, and reversal of the epithelial damage that is observed with PEG-PG/HP-guar thus could also be due to a decrease in the inflammatory response.

Other artificial formulations, such as those containing carboxymethylcellulose and sodium hyaluronate, have also been shown to improve impression cytology grades and reduce squamous metaplasia in keratoconjunctivitis sicca and DED, respectively. ${ }^{22,23}$ Artificial tears are the first choice of treatment and are used in the management of all forms of DED to provide ocular comfort. Overall, these findings suggest that the effect of some artificial tears may extend beyond symptomatic relief and can be beneficial in restoring the ocular surface homeostasis in DED.

This study did not have a specific safety end point; only self-reported or observed AEs were to be recorded. One patient discontinued the study due to an AE; no other AEs were reported. However, the safety of PEG-PG/HP-guar lubricant eye drops has been evaluated in other studies. ${ }^{9,10,12-14}$ These lubricant eye drops are well tolerated with no changes in visual acuity, and no abnormalities reported on slit-lamp examination. ${ }^{9,10,12-14}$

The absence of a control/comparator group, the openlabel design, and a lack of a statistical hypothesis are some of the limitations of this study. In addition, the study did not assess symptomatic improvement in patients which would have been a useful correlation for the change in signs of DED translating into visible improvement in patient's quality of life. Also, though not due to the study criteria, none of the patients in the study had impression cytology $\geq$ Grade 3 ; data from these patients would have provided more supportive evidence of the effect of PEG-PG/HP-guar artificial tears in reversing ocular surface damage/squamous metaplasia even in severe DED. The inclusion of such patients should therefore be considered in future studies. Further, an analysis by age groups would be useful to understand if any difference is observed in the treatment efficacy due to aging effects.

\section{Conclusion}

The PEG-PG/HP-guar artificial tear formulation has a beneficial effect on the tear film stability and ocular health. The PEG-PG/HP-guar artificial tears, used thrice daily for 90 days, reduced squamous metaplasia, in addition to improving TFBUT and corneal staining in patients with DED. Further clinical studies are required to confirm the present findings.

\section{Acknowledgments}

This study was funded by Alcon Research Ltd. (now Novartis Pharmaceutical Corporation), Fort Worth, TX, USA. Medical writing support for this paper was provided by Shivani Vadapalli (Novartis Healthcare Pvt. Ltd., Hyderabad, India).

\section{Disclosure}

The authors report no conflicts of interest in this work.

\section{References}

1. Hodges RR, Dartt DA. Tear film mucins: front line defenders of the ocular surface; comparison with airway and gastrointestinal tract mucins. Exp Eye Res. 2013;117:62-78.

2. Stephens DN, McNamara NA. Altered mucin and glycoprotein expression in dry eye disease. Optom Vis Sci. 2015;92(9):931-938.

3. MaCauley HA, Guasch G. Three cheers for the goblet cell: maintaining homeostasis in mucosal epithelia. Trends Mol Med. 2015;21(8): 492-503.

4. Gipson IK. Goblet cells of the conjunctiva: a review of recent findings. Prog Retin Eye Res. 2016;54:49-63.

5. Bron AJ, de Paiva CS, Chauhan SK, et al. TFOS DEWS II pathophysiology report. Ocul Surf. 2017;15(3):438-510.

6. De Paiva CS, Villarreal AL, Corrales RM, et al. Dry eye-induced conjunctival epithelial squamous metaplasia is modulated by interferon- $\gamma$. Invest Ophthalmol Vis Sci. 2007;48(6):2553-2560.

7. Zhang J, Yan X, Li H. Analysis of the correlations of mucins, inflammatory markers, and clinical tests in dry eye. Cornea. 2013;32(7): 928-932.

8. Murube J, Rivas L. Biopsy of the conjunctiva in dry eye patients establishes a correlation between squamous metaplasia and dry eye clinical severity. Eur J Ophthalmol. 2003;13(3):246-256.

9. Springs C. Novel hydroxypropyl-guar gellable lubricant eye drops for treatment of dry eye. Adv Ther. 2010;27(10):681-690.

10. Benelli U. Systane lubricant eye drops in the management of ocular dryness. Clin Ophthalmol. 2011;5:783-790.

11. Torkildsen G. The effects of lubricant eye drops on visual function as measured by the inter-blink interval visual acuity decay test. Clin Ophthalmol. 2009;3:501-506.

12. Davitt WF, Blooenstein M, Martin A, Christensen MT, Martin AE. Efficacy in patients with dry eye after treatment with a new lubricant eye drop formulation. J Ocul Pharmacol Ther. 2010;26(4):347-353.

13. Kading D. A two-week clinical evaluation of the safety of Systane Ultra in contact lens-wearing patients. Clin Ophthalmol. 2010;4:27-32.

14. McDonald M, Schachet JL, Lievens CW, Kern JR. Systane ${ }^{\circledR}$ Ultra lubricant eye drops for treatment of contact lens-related dryness. Eye Contact Lens. 2014;40(2):106-110.

15. Waduthantri S, Yong SS, Tan CH, Htoon HM, Tong L. Lubricant with gelling agent in treating dry eye in adult Chinese patients. Optom Vis Sci. 2012;89(11):1647-1653.

16. Ubels JL, Clousing DP, Van Haitsma TA, et al. Pre-clinical investigation of the efficacy of an artificial tear solution containing hydroxypropylguar as a gelling agent. Curr Eye Res. 2004;28(6):437-444.

17. Christensen MT, Cohen S, Rinehart J, et al. Clinical evaluation of an HP-guar gellable lubricant eye drop for the relief of dryness of the eye. Curr Eye Res. 2004;28(1):55-62. 
18. Hartstein I, Khwarg S, Przydryga J. An open-label evaluation of HP-Guar gellable lubricant eye drops for the improvement of dry eye signs and symptoms in a moderate dry eye adult population. Curr Med Res Opin. 2005;21(2):255-260.

19. Cervan-Lopez I, Saenz-Frances-San-Baldomero F, Benitez-DelCastillo JM. Reduction of corneal permeability in patients treated with HPGuar: a fluorometric study. Arch Soc Esp Oftalmol. 2006;81:327-332.

20. Fernandez KB, Epstein SP, Raynor GS, et al. Modulation of HLA-DR in dry eye patients following 30 days of treatment with a lubricant eyedrop solution. Clin Ophthalmol. 2015;9:1137-1145.
21. Sánchez MA, Arriola-Villalobos P, Torralbo-Jiménez P, et al. The effect of preservative-free HP-Guar on dry eye after phacoemulsification: a flow cytometric study. Eye (Lond). 2010;24(8):1331-1337.

22. Grene BR, Lankston P, Mordaunt J, Harrold M, Gwon A, Jones R. Unpreserved carboxymethylcellulose artificial tears evaluated in patients with keratoconjunctivitis Sicca. Cornea. 1992;11(4):294-301.

23. Aragona P, Papa V, Micali A, Santocono M, Milazzo G. Long term treatment with sodium hyaluronate-containing artificial tears reduces ocular surface damage in patients with dry eye. Br J Opthalmol. 2001; 86(2):181-184.
Clinical Ophthalmology

\section{Publish your work in this journal}

Clinical Ophthalmology is an international, peer-reviewed journal covering all subspecialties within ophthalmology. Key topics include: Optometry; Visual science; Pharmacology and drug therapy in eye diseases; Basic Sciences; Primary and Secondary eye care; Patien Safety and Quality of Care Improvements. This journal is indexed on

Submit your manuscript here: http://www.dovepress.com/clinical-ophthalmology-journal

\section{Dovepress}

PubMed Central and CAS, and is the official journal of The Society of Clinical Ophthalmology (SCO). The manuscript management system is completely online and includes a very quick and fair peer-review system, which is all easy to use. Visit http://www.dovepress.com/ testimonials.php to read real quotes from published authors. 